Article

\title{
Existence and Integral Representation of Scalar Riemann-Liouville Fractional Differential Equations with Delays and Impulses
}

\author{
Ravi Agarwal ${ }^{1,2}$, Snezhana Hristova ${ }^{3, *}$ (D) and Donal O'Regan ${ }^{4}$ \\ 1 Department of Mathematics, Texas A\&M University-Kingsville, Kingsville, TX 78363, USA; \\ agarwal@tamuk.edu \\ 2 Distinguished University Professor of Mathematics, Florida Institute of Technology, \\ Melbourne, FL 32901, USA \\ 3 Department of Applied Mathematics and Modeling, University of Plovdiv "Paisii Hilendarski", \\ 4000 Plovdiv, Bulgaria \\ 4 School of Mathematics, Statistics and Applied Mathematics, National University of Ireland, \\ H91 TK33 Galway, Ireland; donal.oregan@nuigalway.ie \\ * Correspondence: snehri@gmail.com
}

Received: 26 March 2020; Accepted: 14 April 2020; Published: 16 April 2020

\begin{abstract}
Nonlinear scalar Riemann-Liouville fractional differential equations with a constant delay and impulses are studied and initial conditions and impulsive conditions are set up in an appropriate way. The definitions of both conditions depend significantly on the type of fractional derivative and the presence of the delay in the equation. We study the case of a fixed lower limit of the fractional derivative and the case of a changeable lower limit at each impulsive time. Integral representations of the solutions in all considered cases are obtained. Existence results on finite time intervals are proved using the Banach principle.
\end{abstract}

Keywords: Riemann-Liouville fractional derivative; delay; impulses; initial value problem; existence

\section{Introduction}

The dynamics of many real world processes are adequately modeled by differential equations with impulses and such processes are natural in biology, physics and engineering. The introduction of impulses in fractional calculus might affect the solution's behavior and its integral representation of fractional order. In connection with the memory property of fractional derivatives, in the literature there are mainly two approaches used to introduce impulses to fractional equations: one with a fixed lower limit of the fractional derivative at the initial time and the other with a changeable lower limit of the fractional derivative at each time of impulse. Many results are obtained for Caputo fractional differential equations by applying both approaches for the interpretation of impulses (see, for example, [1-5]). We note in the case of the Caputo fractional derivative there is a similarity of both the initial conditions and the impulsive condition between fractional equations and ordinary equations. However for Riemann-Liouville (RL) fractional differential equations both the initial condition and impulsive conditions have to be appropriately given (which is different than the ordinary case). In [6] the impulsive condition at a point $t_{k}$ is given in the form $\left.t_{k} I_{t}^{1-q} x(t)\right|_{t=t_{k}}-t_{k-1} I_{t}^{1-q} x\left(t_{k}\right)=$ $\phi_{k}\left(x\left(t_{k}\right)\right)$. This form is strange since it connects the values at two consecutive points of impulses and the meaning of the abrupt change occurring at the impulsive point is lost. Impulsive RL fractional differential equations are studied in $[7,8]$ with the impulsive condition at the impulsive point $t_{k}$ given in the form $\left.\left.{ }_{0} I_{t}^{1-q} x(t)\right|_{t=t_{k}+-} I_{t}^{1-q} x(t)\right|_{t=t_{k}-}=\phi_{k}\left(x\left(t_{k}\right)\right)$ with a very complicated definition of a mild 
solution. Impusive RL fractional differential equations are studied in [9] with a very special type of impulsive conditions.

Additionally, the presence of delays in the fractional differential equations can cause several difficulties especially, when the RL fractional derivative is applied. This is connected with the type of the initial condition. In $[10,11]$ the lower bound of the RL fractional derivative coincides with the left side end of the initial interval but we note that this does not correspond to the idea in the case of delay differential equations with ordinary derivatives and the idea of the initial value problem of RL fractional differential equations.

In this paper we study scalar nonlinear RL fractional differential equations with impulses:

- We define the initial conditions and the impulsive conditions based on the physical meaning to initial conditions expressed in terms of Riemann-Liouville fractional derivatives or integrals given by Heymans and Podlubny in [12].

- We set up appropriate initial value problems for delay RL fractional differential equations with the lower limit of the RL derivative equal to the right side end point of the initial interval.

We study two different types of interpretation of the solution of impulsive equations, i.e., fixed lower limit of the RL fractional derivative at the initial time and the RL fractional derivative with changeable lower limit at each impulsive time. Both approaches are applied to set up the impulsive conditions for RL fractional differential equations with impulses in two different ways-the integral form of the impulsive condition and the weighted form of the impulsive condition. Both types of impusive conditions are used to define initial value problems for RL fractional differential equations with a delay and impulses. Integral representations for their solutions are given in all studied cases. The existence and uniqueness is studied.

\section{Preliminary Notes on Fractional Derivatives and Equations}

Let $J=\left(t_{0}, T\right], t_{0} \geq 0, t_{0}<T \leq \infty$ (if $T=\infty$ then the interval $J$ is open). Let $L_{1}^{\text {loc }}(J, \mathbb{R})$ be the linear space of all locally Lebesgue integrable functions $m: J \rightarrow \mathbb{R}^{m}, J \subset \mathbb{R}$. In this paper we will use the following definitions for fractional derivatives and integrals:

- $\quad$ Riemann-Liouville $(R L)$ fractional integral of order $q \in(0,1)([13,14])$

$$
t_{0} I_{t}^{q} m(t)=\frac{1}{\Gamma(q)} \int_{t_{0}}^{t} \frac{m(s)}{(t-s)^{1-q}} d s, \quad t \in J,
$$

where $\Gamma$ is the Gamma function.

- Riemann-Liouville (RL) fractional derivative of order $q \in(0,1)([13,14])$

$$
{ }_{t_{0}}^{R L} D_{t}^{q} m(t)=\frac{d}{d t}\left(t_{0} I_{t}^{1-q} m(t)\right)=\frac{1}{\Gamma(1-q)} \frac{d}{d t} \int_{t_{0}}^{t}(t-s)^{-q} m(s) d s, \quad t \in J .
$$

We will call the point $t_{0}$ a lower limit of the RL fractional derivative.

The definitions of the initial condition for fractional differential equations with RL-derivatives are based on the following result:

Lemma 1 (Lemma $3.2[15])$. Let $q \in(0,1)$ and $m \in L_{1}^{\text {loc }}\left(\left[t_{0}, T\right], \mathbb{R}\right)$.

(a) If there exists a.e. a limit $\lim _{t \rightarrow t_{0}+}\left[\left(t-t_{0}\right)^{1-q} m(t)\right]=C$, then there also exists a limit

$$
\left.t_{0} I_{t}^{1-q} m(t)\right|_{t=t_{0}}:=\lim _{t \rightarrow t_{0}+} t_{0} I_{t}^{1-q} m(t)=C \Gamma(q) .
$$


(b) If there exists a.e. a limit $\left.t_{0} I_{t}^{1-q} m(t)\right|_{t=t_{0}}=B$ and if there exists the limit $\lim _{t \rightarrow t_{0}+}\left[\left(t-t_{0}\right)^{1-q} m(t)\right]$ then

$$
\lim _{t \rightarrow t_{0}+}\left[\left(t-t_{0}\right)^{1-q} m(t)\right]=\frac{B}{\Gamma(q)}
$$

Let $0 \leq a<b \leq \infty$ and consider the scalar RL fractional differential equation

$$
{ }_{a}^{R L} D_{t}^{q} x(t)=f(t, x(t)), \quad t \in(a, b]
$$

where $f:[a, b] \times \mathbb{R} \rightarrow \mathbb{R}$.

Remark 1. Note that according to [15] the initial conditions to (1) could be one of the following forms:

- integral form (see (3.1.6) [15])

$$
\left.{ }_{a} I_{t}^{1-q} x(t)\right|_{t=a}=B, \quad B=\text { constant }
$$

- $\quad$ weighted Cauchy type problem (see and (3.1.7) [15])

$$
\lim _{t \rightarrow a+}\left((t-a)^{1-q} x(t)\right)=C, \quad C=\text { constant }
$$

Remark 2. According to Lemma 1 if the function $x(t)$ satisfies the initial conditions (3), then, $x(t)$ also satisfies the conditions (2) with $B=C \Gamma(q)$.

Proposition 1 (Lemma 5.2 [13]). Suppose the function $f:[a, b] \times \mathbb{R} \rightarrow \mathbb{R}$ is continuous, bounded and Lipschitz with respect to the second variable.

Then the solution of the Cauchy type problem (1), (2) satisfies the Volterra integral equation

$$
x(t)=\frac{B}{\Gamma(q)(t-a)^{1-q}}+\frac{1}{\Gamma(q)} \int_{a}^{t}(t-s)^{q-1} f(s, x(s)) d s, \quad t \in[a, b]
$$

and vise verse.

\section{Interpretations of the Impulses in the RL Fractional Equations}

Let an increasing sequence of non-negative points $\left\{t_{i}\right\}_{i=0}^{\infty}$ be given, $\lim _{k \rightarrow \infty} t_{k}=\infty, \tau>0$ be a given number (it will measure the delay) and $T: t_{0}<T \leq \infty$. Without loss of generality we could assume $T=t_{m+1}$ where $m$ is a natural number (in the case $T=\infty$ we have $m=\infty$ ).

Remark 3. The points $t_{k}, k=1,2, \ldots, m$, are called points of impulses.

We recall the interpretation of the impulses in ordinary differential equations: The impulse at the point $\tilde{t}$ means that there is an instantaneous jump of the solution $x(t)$ from the value $x(\tilde{t}-0)=$ $\lim _{\varepsilon \rightarrow 0+} x(\tilde{t}-\varepsilon)$ before the jump to the value $x(\tilde{t}+0)=\lim _{\mathcal{\varepsilon} \rightarrow 0+} x(\tilde{t}+\varepsilon)$ after the jump and for $t>\tilde{t}$ the solution is determined by the same differential equation but with the new initial value $x(\tilde{t}+0)$.

Now, let us discuss the interpretation of the impulses in the RL fractional differential equations:

1. The significant dependence of the RL derivative on the lower limit (different than the ordinary derivative) leads to two basic types of interpretation:

- $\quad$ Fixed lower limit of the RL fractional derivative - in this case the lower limit of the fractional derivative is kept equal to the initial time over the whole interval of consideration. At points of impulses the amount of jump is taken into account. 
- $\quad$ Changed lower limit of the RL fractional derivative at each time of impulses - each time of impulse is considered as an initial time of the fractional differential equation. Then the lower limit of RL fractional derivative, being equal to the initial time, is changed at each impulsive time.

2. The initial value of the RL fractional equation (see [15]) leads to a non-useful impulsive condition at point $\tilde{t}$ of the type $x(\tilde{t}+0)=G(x(\tilde{t}-0))$. Combining the type of the initial conditions (see Remark 1) and the idea for the meaning of impulses, described above, it leads to two types of impulsive conditions at the point $\tilde{t}$ :

- integral form of the impulsive condition

$$
\left.\tilde{t} I_{t}^{1-q} x(t)\right|_{t=\tilde{t}}=G(x(\tilde{t}-0))
$$

- weighted form of the impulsive condition

$$
\lim _{t \rightarrow \tilde{t}+}\left((t-\tilde{t})^{1-q} x(t)\right)=G(x(\tilde{t}-0))
$$

where $G: \mathbb{R} \rightarrow \mathbb{R}$ is a given function.

Remark 4. Note that for $q \rightarrow 1$ we have $\left.\tilde{t}_{t}^{1-q} x(t)\right|_{t=\tilde{t}}=\frac{\lim _{t \rightarrow \tilde{t}+}\left[(t-\tilde{t})^{1-q} x(t)\right]}{\Gamma(q)} \rightarrow x(\tilde{t}+0)$ and the impulsive condition $\left.\tilde{t}_{t}^{1-q} x(t)\right|_{t=\tilde{t}}=G(x(\tilde{t}-0))$ is reduced to the well known impulsive condition $x(\tilde{t}+0)=G(x(\tilde{t}))$ at the impulsive time $\tilde{t}$ for equations with the ordinary derivative.

In connection with the above descriptions we will introduce the following set

$$
\begin{aligned}
P L_{1}^{l o c}(J, \mathbb{R})= & \left\{u: J \rightarrow \mathbb{R}: u \in L_{1}^{l o c}\left(\cup_{i=0}^{m}\left(t_{k}, t_{k+1}\right), \mathbb{R}\right)\right. \\
& u\left(t_{k}\right)=u\left(t_{k}-0\right)=\lim _{\varepsilon \rightarrow 0+} u\left(t_{k}-\varepsilon\right)<\infty \\
& \left.\left(t-t_{k}\right)^{1-q} u(t) \in C\left(\left(t_{k}, t_{k+1}\right], \mathbb{R}\right) \text { for } k=0,1,2, \ldots, m\right\}
\end{aligned}
$$

For any $k=0,1,2, \ldots, m$ we let $\|u\|_{k+1}=\sup _{t \in\left(t_{k}, t_{k+1}\right]}\left(t-t_{k}\right)^{1-q}|u(t)|$. Then introduce the norm in the set $P L_{1}^{\text {loc }}(J, \mathbb{R})$ defined by $\|u\|_{J}=\max _{k=0,1,2, \ldots, m}\|u\|_{k+1}$.

\section{Integral Representation of the Solution of RL Fractional Differential Equations with Impulses}

We study scalar RL fractional differential equations with impulses and two types of both initial and impulsive conditions. We will apply the two different approaches in the interpretation of the solution of impulsive RL fractional differential equations described in the previous section.

\subsection{Fixed Lower Limit at the Initial Time of RL Fractional Derivative}

\subsubsection{Integral form of the Initial Condition and the Impulsive Conditions}

Consider the scalar Riemann-Liouville delay fractional differential equations with a fixed lower bound of the RL fractional derivative at the given initial time:

$$
{ }_{t_{0}}^{R L} D_{t}^{q} x(t)=f(t, x(t), x(t-\tau)) \text { for } t \in\left(t_{k}, t_{k+1}\right], k=0,1, \ldots, m
$$

with the integral form of the initial condition

$$
x\left(t+t_{0}\right)=\phi(t), t \in[-\tau, 0], \text { and }\left.t_{0} I_{t}^{1-q} x(t)\right|_{t=t_{0}}=\phi(0),
$$


and the integral form of the impulsive condition

$$
\left.t_{k} I_{t}^{1-q} x(t)\right|_{t=t_{k}}=B_{k}\left(x\left(t_{k}-0\right)\right), \text { for } k=1,2, \ldots, m,
$$

where $B_{k} \in C(\mathbb{R}, \mathbb{R}), k=1,2, \ldots, m, \phi \in C([-\tau, 0], \mathbb{R})$ with $|\phi(0)|<\infty, f \in C\left(\left[t_{0}, T\right] \times \mathbb{R} \times \mathbb{R}, \mathbb{R}\right)$.

Theorem 1. Let the following conditions be satisfied:

1. The function $f \in C\left(\left[t_{0}, T\right] \times \mathbb{R} \times \mathbb{R}, \mathbb{R}\right)$ is bounded and there exist constants $L, M>0$ such that $|f(t, x, u)-f(t, y, v)|<L|x-y|+M|u-v|$ for all $t \in\left[t_{0} . T\right], x, y, u, v \in \mathbb{R}$.

2. The function $\phi \in C([-\tau, 0], \mathbb{R}$.

Then the solution $x \in P L_{1}^{\text {loc }}(J, \mathbb{R})$ of the initial value problem for the nonlinear scalar RL fractional delay differential equation with impulses (5)-(7) satisfies

$$
\begin{gathered}
x(t)=\frac{B_{k}\left(x\left(t_{k}\right)\right)}{\Gamma(q)\left(t-t_{k}\right)^{1-q}}+\frac{1}{\Gamma(q)} \int_{t_{k}}^{t} \frac{f(s, x(s), x(s-\tau)))+h_{k}(s)}{(t-s)^{1-q}} d s, \\
\quad \text { for } t \in\left(t_{k}, t_{k+1}\right], k=0,1,2, \ldots, m
\end{gathered}
$$

where $B_{0}(x) \equiv x$,

$$
h_{k}(t)=\frac{q}{\Gamma(1-q)} \int_{t_{0}}^{t_{k}} \frac{x(s)}{(t-s)^{1+q}} d s, t \in\left(t_{k}, t_{k+1}\right], k=1,2, \ldots, m,
$$

and vice verse, i.e., if the function $x:\left[t_{0}-\tau, T\right] \rightarrow \mathbb{R}$ satisfies the integral equalities (8) on $\mathrm{J}$ and $x\left(t+t_{0}\right)=$ $\phi(t), t \in[-\tau, 0]$ then it is a solution of the initial value problem for the nonlinear scalar $R L$ fractional delay differential equation with impulses (5)-(7).

Proof. Let the function $x \in P L_{1}^{\text {loc }}(J, \mathbb{R})$ be a solution of the initial value problem for the nonlinear scalar RL fractional delay differential equation with impulses (5)-(7).

Let $t_{1} \leq t_{0}+\tau$. For $t \in\left[t_{0}, t_{1}\right]$ from Proposition 1 with $a=t_{0}$ and (6) we have

$$
x(t)=\frac{\phi(0)}{\Gamma(q)\left(t-t_{0}\right)^{1-q}}+\frac{1}{\Gamma(q)} \int_{t_{0}}^{t} \frac{f\left(s, x(s), \phi\left(s-\tau-t_{0}\right)\right)}{(t-s)^{1-q}} d s,
$$

i.e., equality (8) holds for $k=0$.

Let $t_{1}>t_{0}+\tau$. Then for $t \in\left[t_{0}, t_{0}+\tau\right]$ the inequality (10) holds. From Proposition 1 with $a=t_{0}, b=t_{1}$ we get for $t \in\left(t_{0}+\tau, t_{1}\right]$

$$
\begin{aligned}
x(t) & =\frac{\phi(0)}{\Gamma(q)\left(t-t_{0}\right)^{1-q}}+\frac{1}{\Gamma(q)} \int_{t_{0}}^{t_{0}+\tau} \frac{f\left(s, x(s), \phi\left(s-\tau-t_{0}\right)\right)}{(t-s)^{1-q}} d s \\
& +\frac{1}{\Gamma(q)} \int_{t_{0}+\tau}^{t} \frac{f(s, x(s), x(s-\tau)}{(t-s)^{1-q}} d s,
\end{aligned}
$$

where $x(s-\tau), s \in\left[t_{0}+\tau, t_{1}\right]$ is defined by (10).

Let $t \in\left(t_{1}, t_{2}\right]$. Then

$$
\begin{aligned}
& { }_{t_{0}}^{R L} D_{t}^{q} x(t)=\frac{1}{\Gamma(1-q)} \frac{d}{d t} \int_{t_{0}}^{t_{1}}(t-s)^{-q} x(s) d s+\frac{1}{\Gamma(1-q)} \frac{d}{d t} \int_{t_{1}}^{t}(t-s)^{-q} x(s) d s \\
& =-h_{1}(t)+{ }_{t_{1}}^{R L} D_{t}^{q} x(t) .
\end{aligned}
$$


From (5) and (7) we get the following initial value problem

$$
\begin{aligned}
& { }_{t_{1}}^{R L} D_{t}^{q} x(t)=F(t, x(t)), \quad t \in\left(t_{1}, t_{2}\right] \\
& \left.t_{1} I_{t}^{1-q} x(t)\right|_{t=t_{1}}=B_{1}\left(x\left(t_{1}-0\right)\right) .
\end{aligned}
$$

where $F(t, x)=f(t, x, x(t-\tau))+h_{1}(t)$ for $t \in\left(t_{1}, t_{2}\right]$ with $x(t-\tau), t \in\left(t_{1}, t_{2}\right]$ and $x\left(t_{1}-0\right)$ defined by (10) and (11).

According to Proposition 1 with $a=t_{1}, b=t_{2}$ we get

$$
x(t)=\frac{B_{1}\left(x\left(t_{1}-0\right)\right)}{\Gamma(q)\left(t-t_{1}\right)^{1-q}}+\frac{1}{\Gamma(q)} \int_{t_{1}}^{t} \frac{f(s, x(s), x(s-\tau))+h_{1}(s)}{(t-s)^{1-q}} d s, t \in\left(t_{1}, t_{2}\right],
$$

i.e., (8) holds for $k=1$.

Let $t \in\left(t_{2}, t_{3}\right]$. Then

$$
\begin{aligned}
& { }_{t_{0}}^{R L} D_{t}^{q} x(t)=\frac{1}{\Gamma(1-q)} \frac{d}{d t} \int_{t_{0}}^{t_{2}}(t-s)^{-q} x(s) d s+\frac{1}{\Gamma(1-q)} \frac{d}{d t} \int_{t_{2}}^{t}(t-s)^{-q} x(s) d s \\
& =-h_{2}(t)+{ }_{t_{2}}^{R L} D_{t}^{q} x(t) .
\end{aligned}
$$

From (5), (15) and (7) we get the following initial value problem

$$
\begin{aligned}
& { }_{t_{2}}^{R L} D_{t}^{q} x(t)=F(t, x(t)), \quad t \in\left(t_{2}, t_{3}\right] \\
& \left.t_{2} I_{t}^{1-q} x(t)\right|_{t=t_{2}}=B_{2}\left(x\left(t_{2}-0\right)\right)
\end{aligned}
$$

where $F(t, x)=f(t, x, x(t-\tau))+h_{2}(t)$ with $x(t-\tau), t \in\left(t_{2}, t_{3}\right]$ and $x\left(t_{2}-0\right)$ defined by (14)

According to Proposition 1 with $a=t_{2}, b=t_{3}$ we obtain

$$
x(t)=\frac{B_{2}\left(x\left(t_{2}-0\right)\right)}{\Gamma(q)\left(t-t_{2}\right)^{1-q}}+\frac{1}{\Gamma(q)} \int_{t_{2}}^{t} \frac{f(s, x(s), x(s-\tau))+h_{2}(s)}{(t-s)^{1-q}} d s, \quad t \in\left(t_{2}, t_{3}\right]
$$

i.e., (8) holds for $k=2$.

Continue this process to prove the integral presentation (8).

Now, let the function $x:\left[t_{0}-\tau, T\right] \rightarrow \mathbb{R}$ satisfies the integral equalities (8) on $J$ and $x\left(t+t_{0}\right)=$ $\phi(t), t \in[-\tau, 0]$. From conditions 1,2 and Equation (8) it follows $x \in P L_{1}^{l o c}(J, \mathbb{R})$.

Let $t \in\left(t_{k}, t_{k+1}\right]$. Then applying $t_{k} I_{t}^{1-q}\left(t-t_{k}\right)^{q-1}=\Gamma(q)$ we get

$$
\begin{aligned}
& \left.t_{k} I_{t}^{1-q} x(t)\right|_{t=t_{k}}=\lim _{t \rightarrow t_{k}+} t_{k} I_{t}^{1-q} x(t) \\
& =\lim _{t \rightarrow t_{k}+}\left\{\frac { 1 } { \Gamma ( 1 - q ) } \int _ { t _ { k } } ^ { t } \frac { 1 } { ( t - s ) ^ { q } } \left(\frac{B_{k}\left(x\left(t_{k}\right)\right)}{\Gamma(q)\left(s-t_{k}\right)^{1-q}}\right.\right. \\
& \left.\left.\quad+\frac{1}{\Gamma(q)} \int_{t_{k}}^{s} \frac{f(\sigma, x(\sigma), x(\sigma-\tau)))+h_{k}(\sigma)}{(s-\sigma)^{1-q}} d \sigma\right) d s\right\} \\
& =\frac{B_{k}\left(x\left(t_{k}\right)\right)}{\Gamma(q)} \lim _{t \rightarrow t_{k}+} t_{k} I_{t}^{1-q}\left(t-t_{k}\right)^{q-1} \\
& \quad+\frac{1}{\Gamma(1-q) \Gamma(q)} \lim _{t \rightarrow t_{k}+}\left(\int_{t_{k}}^{t} \frac{1}{(t-s)^{q}} \int_{t_{k}}^{s} \frac{f(\sigma, x(\sigma), x(\sigma-\tau)))+h_{k}(\sigma)}{(s-\sigma)^{1-q}} d \sigma d s\right) \\
& =B_{k}\left(x\left(t_{k}\right)\right),
\end{aligned}
$$


and from the equality $\frac{d}{d t} \int_{a}^{t}(t-s)^{-q}(s-a)^{q-1} d s=0$ we obtain

$$
\begin{aligned}
{ }_{t_{0}}^{R L} D_{t}^{q} x(t)=\frac{1}{\Gamma(1-q)} \frac{d}{d t} \int_{t_{0}}^{t}(t-s)^{-q} x(s) d s \\
=-\frac{q}{\Gamma(1-q)} \int_{t_{0}}^{t_{k}}(t-s)^{-1-q} x(s) d s+\frac{1}{\Gamma(1-q)} \frac{d}{d t} \int_{t_{k}}^{t}(t-s)^{-q} x(s) d s \\
=-h_{k}(t)+\frac{1}{\Gamma(1-q)} \frac{d}{d t} \int_{t_{k}}^{t}(t-s)^{-q} x(s) d s \\
=-h_{k}(t)+\frac{1}{\Gamma(1-q)}\left(\frac{B_{k}\left(x\left(t_{k}\right)\right)}{\Gamma(q)} \frac{d}{d t} \int_{t_{k}}^{t}(t-s)^{-q} \frac{1}{\left(s-t_{k}\right)^{1-q}} d s\right. \\
\left.+\frac{d}{d t} \int_{t_{k}}^{t}(t-s)^{-q} \frac{1}{\Gamma(q)} \int_{t_{k}}^{s} \frac{f(\sigma, x(\sigma), x(\sigma-\tau)))+h_{k}(\sigma)}{(s-\sigma)^{1-q}} d \sigma\right) d s \\
\left.=-h_{k}(t)+\frac{1}{\Gamma(1-q)} \frac{d}{d t} \int_{t_{k}}^{t}(t-s)^{-q} \frac{1}{\Gamma(q)} \int_{t_{k}}^{s} \frac{f(\sigma, x(\sigma), x(\sigma-\tau)))+h_{k}(\sigma)}{(s-\sigma)^{1-q}} d \sigma\right) d s \\
=-h_{k}(t)+\frac{1}{\Gamma(1-q)} \frac{d}{d t} \int_{t_{k}}^{t}(t-s)^{-q}\left(t_{k} I_{s}^{q} f(\sigma, x(\sigma), x(\sigma-\tau))+t_{k} I_{s}^{q} h_{k}(s)\right) d s \\
=-h_{k}(t)+{ }_{t_{k}}^{R L} D_{t}^{q}\left(t_{k} I_{t}^{q} f(t, x(t), x(t-\tau))+t_{k} I_{t}^{q} h_{k}(t)\right)=f(t, x(t), x(t-\tau)) .
\end{aligned}
$$

Also,

$$
\begin{aligned}
& \lim _{t \rightarrow t_{0}+}\left(t_{0} I_{t}^{1-q} x(t)\right)=\frac{\phi(0)}{\Gamma(q)} \lim _{t \rightarrow t_{0}+} t_{0} I_{t}^{1-q}\left(t-t_{0}\right)^{q-1} \\
& \quad+\lim _{t \rightarrow t_{0}+} t_{0} I_{t}^{1-q} \frac{1}{\Gamma(q)} \int_{t_{0}}^{s} \frac{f(\sigma, x(\sigma), x(\sigma-\tau))}{(s-\sigma)^{1-q}} d \sigma=\phi(0) .
\end{aligned}
$$

Therefore the function $x(t)$ is a solution of the initial value problem (5)-(7).

\subsubsection{Weighted form of the Initial Condition and the Impulsive Conditions}

Consider the scalar impulsive Riemann-Liouville delay fractional differential equations with a fixed lower bound of the RL fractional derivative at the given initial time (5) with weighted form of the initial condition

$$
x\left(t+t_{0}\right)=\phi(t), t \in[-\tau, 0], \text { and } \lim _{t \rightarrow t_{0}+}\left(\left(t-t_{0}\right)^{1-q} x(t)\right)=\phi(0),
$$

and weighted form of the impulsive condition

$$
\lim _{t \rightarrow t_{k}+}\left(\left(t-t_{k}\right)^{1-q} x(t)\right)=B_{k}\left(x\left(t_{k}-0\right)\right), \quad \text { for } k=1,2, \ldots, m
$$

where $B_{k} \in C(\mathbb{R}, \mathbb{R}), k=1,2, \ldots, \phi \in C([-\tau, 0], \mathbb{R})$.

From Theorem 1 and Lemma 1 (a) the following result follows for the weighted form of both the initial condition and the impulsive condition: 
Theorem 2. The solution $x \in P L_{1}^{l o c}(J, \mathbb{R})$ of the initial value problem for the nonlinear scalar $R L$ fractional delay differential equation with impulses (5), (19), (20) satisfies

$$
\begin{gathered}
x(t)=\frac{B_{k}\left(x\left(t_{k}-0\right)\right)}{\left(t-t_{k}\right)^{1-q}}+\frac{1}{\Gamma(q)} \int_{t_{k}}^{t} \frac{f(s, x(s), x(s-\tau)))+h_{k}(s)}{(t-s)^{1-q}} d s, \\
\quad \text { for } t \in\left(t_{k}, t_{k+1}\right], k=0,1,2, \ldots, m
\end{gathered}
$$

where $B_{0}(x) \equiv x, h_{k}(t)$ is defined by (9) and vice verse, i.e., if the function $x:\left[t_{0}-\tau, T\right] \rightarrow \mathbb{R}$ satisfies the integral equalities (21) on $J$ and $x\left(t+t_{0}\right)=\phi(t), t \in[-\tau, 0]$ then it is a solution of the initial value problem for the nonlinear scalar RL fractional delay differential equation with impulses (5), (19), (20).

\subsection{Changed Lower Limit of the RL Fractional Derivative at Each Impulsive Time}

\subsubsection{Integral form of the Initial Condition and the Impulsive Conditions}

Consider the scalar Riemann-Liouville delay fractional differential equations with a changeable lower bound of the RL fractional derivative at any impulsive time :

$$
{ }_{t_{k}}^{R L} D_{t}^{q} x(t)=f(t, x(t), x(t-h)) \text { for } t \in\left(t_{k}, t_{k+1}\right], k=0,1, \ldots, m
$$

with the integral form of the initial condition (6) and the integral form of the impulsive condition (7) where $B_{k} \in C(\mathbb{R}, \mathbb{R}), k=1,2, \ldots, m, \phi \in C([-\tau, 0], \mathbb{R}), f \in C(J \times \mathbb{R} \times \mathbb{R}, \mathbb{R})$.

Theorem 3. Let the conditions of Theorem 1 be satisfied. Then the solution $x \in P L_{1}^{\text {loc }}(J, \mathbb{R})$ of the initial value problem for the nonlinear scalar RL fractional delay differential equation with impulses (22), (6), (7) satisfies

$$
\begin{gathered}
x(t)=\frac{B_{k}\left(x\left(t_{k}\right)\right)}{\Gamma(q)\left(t-t_{k}\right)^{1-q}}+\frac{1}{\Gamma(q)} \int_{t_{k}}^{t} \frac{f(s, x(s), x(s-\tau)))}{(t-s)^{1-q}} d s, \\
\quad \text { for } t \in\left(t_{k}, t_{k+1}\right], k=0,1,2, \ldots, m
\end{gathered}
$$

where $B_{0}(x) \equiv x$ and vice verse, i.e., if the function $x:\left[t_{0}-\tau, T\right] \rightarrow \mathbb{R}$ satisfies the integral equalities (23) on $J$ and $x\left(t+t_{0}\right)=\phi(t), t \in[-\tau, 0]$ then it is a solution of the initial value problem for the nonlinear scalar $R L$ fractional delay differential equation with impulses (22), (6), (7).

Proof. Let the function $x \in P L_{1}^{l o c}(J, \mathbb{R})$ be a solution of the initial value problem for the nonlinear scalar RL fractional delay differential equation with impulses (22), (6), (7). Using induction for any integer $k=0,1,2, \ldots, m$ and $t \in\left(t_{k}, t_{k+1}\right]$ it satisfies the integral equality (23).

Let the function $x \in P L_{1}^{\text {loc }}(J, \mathbb{R})$ satisfies the integral equality (23).

Let $t \geq t_{0}$ be such that $t \in\left(t_{k}, t_{k+1}\right]$ for $k=0,1, \ldots, m$. Then applying $t_{k} I_{t}^{1-q}\left(t-t_{k}\right)^{q-1}=\Gamma(q)$ we get

$$
\begin{aligned}
& \left.t_{k} I_{t}^{1-q} x(t)\right|_{t=t_{k}}=\frac{B_{k}\left(x\left(t_{k}-0\right)\right)}{\Gamma(q)} \lim _{t \rightarrow t_{k}+}{ }_{t_{k}} I_{t}^{1-q}\left(t-t_{k}\right)^{q-1} \\
& +\frac{1}{\Gamma(1-q) \Gamma(q)} \lim _{t \rightarrow t_{k}+}\left(\int_{t_{k}}^{t} \frac{1}{(t-s)^{q}} \int_{t_{k}}^{s} \frac{f(\sigma, x(\sigma), x(\sigma-\tau)))+h_{k}(\sigma)}{(s-\sigma)^{1-q}} d \sigma d s\right) \\
& =B_{k}\left(x\left(t_{k}-0\right)\right),
\end{aligned}
$$


and from the equality $\frac{d}{d t} \int_{a}^{t}(t-s)^{-q}(s-a)^{q-1} d s=0$ we get

$$
\begin{aligned}
& { }_{t_{k}}^{R L} D_{t}^{q} x(t)=\frac{1}{\Gamma(1-q)} \frac{d}{d t} \int_{t_{k}}^{t}(t-s)^{-q} x(s) d s \\
& =\frac{1}{\Gamma(1-q)}\left(\frac{B_{k}\left(x\left(t_{k}-0\right)\right)}{\Gamma(q)} \frac{d}{d t} \int_{t_{k}}^{t}(t-s)^{-q} \frac{1}{\left(s-t_{k}\right)^{1-q}} d s\right. \\
& \left.+\frac{d}{d t} \int_{t_{k}}^{t}(t-s)^{-q} \frac{1}{\Gamma(q)} \int_{t_{k}}^{s} \frac{f(\sigma, x(\sigma), x(\sigma-\tau)))}{(s-\sigma)^{1-q}} d \sigma\right) d s \\
& \left.=\frac{1}{\Gamma(1-q)} \frac{d}{d t} \int_{t_{k}}^{t}(t-s)^{-q} \frac{1}{\Gamma(q)} \int_{t_{k}}^{s} \frac{f(\sigma, x(\sigma), x(\sigma-\tau)))}{(s-\sigma)^{1-q}} d \sigma\right) d s \\
& =\frac{1}{\Gamma(1-q)} \frac{d}{d t} \int_{t_{k}}^{t}(t-s)^{-q}{ }_{t_{k}} I_{s}^{q} f(\sigma, x(\sigma), x(\sigma-\tau)) d s \\
& ={ }_{t_{k}}^{R L} D_{t}^{q} t_{k} I_{t}^{q} f(t, x(t), x(t-\tau))=f(t, x(t), x(t-\tau)) .
\end{aligned}
$$

Also,

$$
\begin{aligned}
& \lim _{t \rightarrow t_{0}+}\left(t_{0} I_{t}^{1-q} x(t)\right)=\frac{x\left(t_{0}\right)}{\Gamma(q)} \lim _{t \rightarrow t_{0}+} t_{0} I_{t}^{1-q}\left(t-t_{0}\right)^{q-1} \\
& \quad+\lim _{t \rightarrow t_{0}+} t_{0} I_{t}^{1-q} \frac{1}{\Gamma(q)} \int_{t_{0}}^{s} \frac{f(\sigma, x(\sigma), x(\sigma-\tau))}{(s-\sigma)^{1-q}} d \sigma=\phi(0) .
\end{aligned}
$$

Therefore the function $x(t)$ is a solution of the initial value problem (22), (6), (7).

\subsubsection{Weighted form of the Initial Condition and the Impulsive Conditions}

Consider the scalar Riemann-Liouville delay fractional differential equations with a fixed lower bound of the RL fractional derivative at the given initial time (22) with weighted form of the initial condition (19) and the weighted form of the impulsive condition (20).

From Theorem 5 and Lemma 1 (a) the following result follows for the weighted form of both the initial condition and the impulsive condition:

Theorem 4. Let the conditions of Theorem 1 be satisfied. Then the solution $x \in P L_{1}^{\text {loc }}(J, \mathbb{R})$ of the initial value problem for the nonlinear scalar RL fractional delay differential equation with impulses (22), (19), (20) satisfies

$$
\begin{gathered}
x(t)=\frac{B_{k}\left(x\left(t_{k}-0\right)\right)}{\left(t-t_{k}\right)^{1-q}}+\frac{1}{\Gamma(q)} \int_{t_{k}}^{t} \frac{f(s, x(s), x(s-\tau)))}{(t-s)^{1-q}} d s, \\
\quad \text { for } t \in\left(t_{k}, t_{k+1}\right], k=0,1,2, \ldots, m
\end{gathered}
$$

where $B_{0}(x) \equiv x$ and vice verse, i.e., if the function $x(t):\left[t_{0}-\tau, T\right] \rightarrow \mathbb{R}$ satisfies the integral equalities (26) on $J$ and $x\left(t+t_{0}\right)=\psi(t), t \in[-\tau, 0]$ then it is a solution of the initial value problem for the nonlinear scalar RL fractional delay differential equation with impulses (22), (19), (20).

\subsection{Fixed Lower Limit vs. Changeable Lower Limit}

Now we will compare formulas (8) and (23). Note both formulas coincide iff $h_{k}(t) \equiv 0$, i.e., in the case of zero solution.

Remark 5. Note that both formulas (8) and (23) are applied step by step w.r.t. the intervals $\left(t_{k}, t_{k+1}\right], k=$ $0,1, \ldots, m$.

Now we will consider a particular example. Let $q=0.5, \tau=1, t_{k}=2 k, k=0,1,2, \ldots, \phi(t)=1$ for $t \in[-1,0], B_{k}(x)=2 x$ and $f(t, x, y)=y$. 
We will calculate the solution in both cases-when the lower limit of RL fractional derivative is fixed at 0 , and when the lower limit of RL fractional integral equals to the impulsive time. The calculations are done using CAS Wolfram Mathematica.

Case 1. (Fixed lower limit of $R L$ fractional derivative at 0 .)

For $t \in(0,1]$ Equation (8) gives the solution

$$
x(t)=\frac{1}{\sqrt{\pi t}}+\frac{1}{\sqrt{\pi}} \int_{0}^{t} \frac{1}{(t-s)^{0.5}} d s=\frac{1}{\sqrt{\pi t}}(1+2 t) .
$$

For $t \in(1,2]$ we get

$$
\begin{aligned}
x(t) & =\frac{1}{\sqrt{\pi t}}+\frac{1}{\sqrt{\pi}} \int_{0}^{1} \frac{1}{(t-s)^{0.5}} d s+\frac{1}{\sqrt{\pi}} \int_{1}^{t} \frac{x(s-1)}{(t-s)^{0.5}} d s \\
& =\frac{1}{\sqrt{\pi t}}+\frac{1}{\sqrt{\pi}}(2 \sqrt{t-1}+2 \sqrt{t})+\frac{1}{\pi} \int_{1}^{t} \frac{2 s-1}{\sqrt{(s-1)(t-s)}} d s \\
& =\frac{1}{\sqrt{\pi t}}+\frac{1}{\sqrt{\pi}}(2 \sqrt{t-1}+2 \sqrt{t})+t=\frac{1}{\sqrt{\pi t}}\left(1+2 \sqrt{t^{2}-t}+2 t\right)+t .
\end{aligned}
$$

Then we obtain

$$
\begin{aligned}
h_{1}(t)= & \frac{0.5}{\Gamma(0.5)} \int_{0}^{2} \frac{x(s)}{(t-s)^{1.5}} d s \\
= & \frac{1}{2 \pi} \int_{0}^{1} \frac{1}{\sqrt{s}(1+2 s)} \frac{1}{(t-s)^{1.5}} d s+\frac{1}{2 \pi} \int_{1}^{2} \frac{\frac{1}{\sqrt{s}}\left(1+2 \sqrt{s^{2}-s}+2 s\right)+s}{(t-s)^{1.5}} d s \\
= & \frac{1}{2 \pi}\left(\int_{0}^{2} \frac{1}{\sqrt{s}(t-s)^{1.5}} d s+\int_{0}^{2} \frac{2 \sqrt{s}}{(t-s)^{1.5}} d s+\int_{1}^{2} \frac{2 \sqrt{s-1}}{(t-s)^{1.5}} d s+\int_{1}^{2} \frac{s}{(t-s)^{1.5}} d s\right) \\
= & \left.\frac{\sqrt{2}}{\pi t \sqrt{t-2}+1.88561}{ }_{2} F_{1}\left[1.5,1.5,2.5, \frac{2}{t}\right]\right) \frac{1}{\pi t \sqrt{t}} \\
& +\frac{2}{3 \pi(t-1) \sqrt{t-1}}{ }_{2} F_{1}\left[1.5,1.5,2.5, \frac{1}{t-1}\right]+\frac{(3-2 t) t-1}{\pi(t-1) \sqrt{t-1}}+\frac{4+t(2 t-6)}{\pi(t-2) \sqrt{t-2}}, \\
& \quad \text { for } t \in(2,4],
\end{aligned}
$$

where ${ }_{2} F_{1}[a, b, c, z]$ is the hypergeometric function.

Then

$$
\begin{aligned}
x(t)= & \frac{\sqrt{\pi} x(2)}{\sqrt{\pi(t-2)}}+\frac{1}{\sqrt{\pi}} \int_{2}^{t} \frac{x(s-1)}{(t-s)^{0.5}} d s+\frac{1}{\sqrt{\pi}} \int_{2}^{t} \frac{h_{1}(s)}{(t-s)^{0.5}} d s \\
= & \frac{5+2 \sqrt{2}(1+\sqrt{\pi})}{\sqrt{2 \pi(t-2)}}+\frac{8 t \sqrt{t}}{3 \pi}{ }_{2} F_{1}[0.5,2,2.5, t]+2(t-2)+\frac{2}{3 \sqrt{\pi}}(2 t-1) \sqrt{t-2} \\
& +\frac{1}{\sqrt{\pi t}}+\frac{2}{t \sqrt{\pi}} \sqrt{t-2}{ }_{2} F_{1}\left[1,1.5,2, \frac{2}{t}\right]+\frac{\sqrt{t-2}}{\sqrt{\pi}(t-1)}{ }_{2} F_{1}\left[1,1.5,2, \frac{2}{t-1}\right] \\
& +\frac{1}{t \pi \sqrt{\pi}} \sqrt{t-2}\left(-0.353553 F_{1}\left[2,1,0.5,3, \frac{1}{t}, 0.5\right]+3.7712361_{2} F_{1}\left[1,2,2.5, \frac{2}{t}\right]\right. \\
& \quad \text { for } t \in(2,3] .
\end{aligned}
$$

Case 2. (Changeable lower limit of $R L$ fractional derivative at the impulsive times $2 k$ ).

For $t \in(0,2]$ the solution is defined by (27) and (28). 
For $t \in(2,3]$ we get

$$
\begin{aligned}
x(t)= & \frac{\sqrt{\pi} x(2)}{\sqrt{\pi(t-2)}}+\frac{1}{\sqrt{\pi}} \int_{2}^{t} \frac{x(s-1)}{(t-s)^{0.5}} d s, \\
= & \frac{\frac{1}{\sqrt{2 \pi}}(5+2 \sqrt{2})+2}{\sqrt{t-2}}+\frac{1}{\pi} \int_{2}^{t} \frac{1}{\sqrt{(s-1)}(t-s)^{0.5}} d s+\frac{1}{\pi} \int_{2}^{t} \frac{2 \sqrt{(s-1)-1}}{(t-s)^{0.5}} d s \\
& +\frac{2}{\pi} \int_{2}^{t} \frac{\sqrt{s-2}}{(t-s)^{0.5}} d s+\frac{1}{\pi} \int_{2}^{t} \frac{2 s-1}{\sqrt{s-1}(t-s)^{0.5}} d s+\frac{1}{\sqrt{\pi}} \int_{2}^{t} \frac{s-1}{(t-s)^{0.5}} d s \\
= & \frac{5+2 \sqrt{2}(1+\sqrt{\pi})}{\sqrt{2 \pi(t-2)}}+\frac{8 t \sqrt{t}}{3 \pi}{ }_{2} F_{1}[0.5,2,2.5, t]+2(t-2)+\frac{2}{3 \sqrt{\pi}}(2 t-1) \sqrt{t-2} .
\end{aligned}
$$

As it is seen from the example, both solutions coincide in the interval $[-1,2]$ where $t_{1}=2$ is the first time of impulse. For $t>2$ both solutions differ (see (30) and (31)).

\section{Existence of the Solution}

Based on Lemma 1 we will study only the RL fractional differential equation with the integral form of the impulsive conditions and the integral form of the initial condition.

We will consider the case $T<\infty$. Without loss of generality we assume there exists a natural number $m$ with $T=t_{m+1}, m<\infty$.

\subsection{Existence for the IVP (5), (6), (7) with Fixed Lower Limit of RL Fractional Derivative}

We consider the following conditions:

(A1) The functions $B_{k} \in C(\mathbb{R}, \mathbb{R}), k=1,2, \ldots, m$ and there exists a constant $C_{k}>0$ such that $\left|B_{k}(x)-B_{k}(y)\right| \leq C_{k}|x-y|$ for $x, y \in \mathbb{R}$.

(A2) The function $f \in C\left(\left[t_{0}, T\right] \times \mathbb{R} \times \mathbb{R}, \mathbb{R}\right)$ is bounded and there exist constants $L, M>0$ such that $|f(t, x, u)-f(t, y, v)| \leq L|x-y|+M|u-v|$ for all $t \in\left[t_{0}, T\right], x, y, u, v \in \mathbb{R}$.

Theorem 5. Let the conditions (A1), (A2) be satisfied on $J=\left[t_{0}, T\right]$ and the inequality

$$
\begin{aligned}
\rho & =\max _{k=1,2, \ldots, m}\left\{\frac{C_{k}}{\Gamma(q)\left(t_{k}-t_{k-1}\right)^{1-q}}+\frac{L\left(t_{k+1}-t_{k}\right)^{q} \Gamma(q)}{\Gamma(2 q)}+\frac{M\left(t_{k+1}-t_{k}\right)}{\Gamma(1+q)\left(t_{k}-\tau-t_{0}\right)^{1-q}}\right. \\
& \left.+\left(\frac{t_{k+1}-t_{k}}{t_{k}-t_{0}}\right)^{1-q} \sum_{i=0}^{k-2} \frac{\Gamma(q)}{\left(t_{k}-t_{i}\right)^{q}}+\left(\frac{t_{k+1}-t_{k}}{t_{k}-t_{k-1}}\right)^{1-q} \frac{\Gamma(q)}{\left(t_{k}-t_{k-1}\right)^{q}}\right\}<1
\end{aligned}
$$

holds.

Then the IVP (5)-(7) has a unique solution $x \in P L^{\text {loc }}(J, \mathbb{R})$.

Proof. Existence. Define the operator $\Xi: P L^{l o c}(J, \mathbb{R}) \rightarrow P L^{l o c}(J, \mathbb{R})$ by

$$
\Xi(x(t))=\left\{\begin{array}{l}
\phi(t) \quad t \in[-\tau, 0] \\
\frac{B_{k}\left(x\left(t_{k}\right)\right)}{\Gamma(q)\left(t-t_{k}\right)^{1-q}}+\frac{1}{\Gamma(q)} \int_{t_{k}}^{t} \frac{f(s, x(s), x(s-\tau)))+H_{k}(s, x)}{(t-s)^{1-q}} d s, \\
\quad \text { for } t \in\left(t_{k}, t_{k+1}\right], k=0,1,2, \ldots, m
\end{array}\right.
$$

where $H_{0}(t, x) \equiv 0$ and $H_{k}(t, x):\left(t_{k}, t_{k+1}\right] \times P L^{\text {loc }}(J, \mathbb{R}) \rightarrow \mathbb{R}$ are defined by

$$
H_{k}(t, x)=\frac{q}{\Gamma(1-q)} \int_{t_{0}}^{t_{k}} \frac{x(s)}{(t-s)^{1+q}} d s, t \in\left(t_{k}, t_{k+1}\right], k=1,2, \ldots, m .
$$


Let $z, y \in P C(J, \mathbb{R})$. Then for $t>t_{i+1}, i=0,1,2, \ldots, m$ we have

$$
\frac{q}{\Gamma(1-q)} \int_{t_{i}}^{t_{i+1}} \frac{\left(s-t_{i}\right)^{1-q}|z(s)-y(s)|}{\left(s-t_{i}\right)^{1-q}(t-s)^{1+q}} d s=\frac{\|z-y\|_{i+1}}{\Gamma(1-q)} \frac{\left(t_{i+1}-t_{i}\right)^{q}}{\left(t-t_{i+1}\right)^{q}\left(t-t_{i}\right)} .
$$

Then from (34) with $i=0$ we obtain

$$
\left|H_{1}(t, z)-H_{1}(t, y)\right|=\frac{\|z-y\|_{1}}{\Gamma(1-q)} \frac{\left(t_{1}-t_{0}\right)^{q}}{\left(t-t_{1}\right)^{q}\left(t-t_{0}\right)}, t \in\left(t_{1}, t_{2}\right]
$$

and

$$
\begin{aligned}
\int_{t_{1}}^{t} \frac{\left|H_{1}(s, z(s))-H_{1}(s, y(s))\right|}{(t-s)^{1-q}} d s & \leq \frac{\|z-y\|_{1}}{\Gamma(1-q)} \int_{t_{1}}^{t} \frac{\left(t_{1}-t_{0}\right)^{q}}{\left(s-t_{1}\right)^{q}(t-s)^{1-q}\left(s-t_{0}\right)} d s \\
& \leq \frac{\Gamma(q)}{\left(t-t_{0}\right)^{1-q}\left(t_{1}-t_{0}\right)^{q}}\|z-y\|_{J}, t \in\left(t_{1}, t_{2}\right] .
\end{aligned}
$$

For $t \in\left(t_{2}, t_{3}\right]$ applying (34) with $i=1$ and $i=2$ we get

$$
\begin{aligned}
& \left|H_{2}(t, z)-H_{2}(t, y)\right|=\frac{q}{\Gamma(1-q)} \int_{t_{0}}^{t_{k}} \frac{|z(s)-y(s)|}{(t-s)^{1+q}} d s \\
& =\frac{q}{\Gamma(1-q)} \int_{t_{0}}^{t_{1}} \frac{|z(s)-y(s)|}{(t-s)^{1+q}} d s+\frac{q}{\Gamma(1-q)} \int_{t_{1}}^{t_{2}} \frac{|z(s)-y(s)|}{(t-s)^{1+q}} d s \\
& \leq \frac{\|z-y\|_{1}}{\Gamma(1-q)} \frac{\left(t_{1}-t_{0}\right)^{q}}{\left(t-t_{1}\right)^{q}\left(t-t_{0}\right)}+\frac{|| z-y \|_{2}}{\Gamma(1-q)} \frac{\left(t_{2}-t_{1}\right)^{q}}{\left(t-t_{2}\right)^{q}\left(t-t_{1}\right)} .
\end{aligned}
$$

and

$$
\begin{aligned}
& \int_{t_{2}}^{t} \frac{\left|H_{2}(s, z(s))-H_{2}(s, y(s))\right|}{(t-s)^{1-q}} d s \\
& \leq \frac{\|z-y\|_{J}}{\Gamma(1-q)}\left(\int_{t_{2}}^{t} \frac{1}{\left(s-t_{1}\right)^{q}(t-s)^{1-q}\left(s-t_{0}\right)} d s+\int_{t_{2}}^{t} \frac{1}{\left(s-t_{2}\right)^{q}(t-s)^{1-q}\left(s-t_{1}\right)} d s\right) \\
& \leq \frac{\|z-y\|_{J}}{\Gamma(1-q)}\left(\int_{t_{2}}^{t} \frac{1}{\left(s-t_{2}\right)^{q}(t-s)^{1-q}\left(s-t_{0}\right)} d s+\int_{t_{2}}^{t} \frac{1}{\left(s-t_{2}\right)^{q}(t-s)^{1-q}\left(s-t_{1}\right)} d s\right) \\
& \leq\left(\frac{1}{\left(t-t_{0}\right)^{1-q}\left(t_{2}-t_{0}\right)^{q}}+\frac{1}{\left(t-t_{1}\right)^{1-q}\left(t_{2}-t_{1}\right)^{q}}\right) \Gamma(q)\|z-y\|_{J}, t \in\left(t_{2}, t_{3}\right] .
\end{aligned}
$$

By the induction process we obtain

$$
\begin{aligned}
& \int_{t_{k}}^{t} \frac{\left|H_{k}(s, z(s))-H_{k}(s, y(s))\right|}{(t-s)^{1-q}} d s \\
& \leq \Gamma(q)\|z-y\|_{J}\left(\frac{1}{\left(t-t_{0}\right)^{1-q}} \sum_{i=0}^{k-2} \frac{1}{\left(t_{k}-t_{i}\right)^{q}}+\frac{1}{\left(t_{k}-t_{k-1}\right)^{q}\left(t-t_{k-1}\right)^{1-q}}\right), \quad t \in\left(t_{k}, t_{k+1}\right] .
\end{aligned}
$$


Let $t \in\left(t_{0}, t_{1}\right]$. Then we obtain

$$
\begin{aligned}
|\Xi(z(t))-\Xi(y(t))| & \leq \frac{1}{\Gamma(q)} \int_{t_{0}}^{t} \frac{|f(s, z(s), z(s-\tau))-f(s, y(s), y(s-\tau))|}{(t-s)^{1-q}} d s, \\
& \leq L \frac{1}{\Gamma(q)} \int_{t_{0}}^{t}(t-s)^{q-1}\left(s-t_{0}\right)^{q-1} d s\|z-y\|_{J} \\
& =L \frac{\left(t-t_{0}\right)^{2 q-1} \Gamma(q)}{\Gamma(2 q)}\|z-y\|_{J} \\
& \leq L\left(t_{1}-t_{0}\right)^{q} \frac{\left(t-t_{0}\right)^{q-1} \Gamma(q)}{\Gamma(2 q)}\|z-y\|_{J} .
\end{aligned}
$$

and

$$
\left(t-t_{0}\right)^{1-q}|\Xi(z(t))-\Xi(y(t))| \leq L \frac{\left(t_{1}-t_{0}\right)^{q} \Gamma(q)}{\Gamma(2 q)}\|z-y\|_{1} .
$$

Let $t \in\left(t_{k}, t_{k+1}\right], k=1,2, \ldots, m$. If $t_{k} \leq t_{0}+\tau$ then the inequality (40) holds. Let $t_{k}>t_{0}+\tau$. Then for any $s \in\left[t_{k}, t\right], t \in\left(t_{k}, t_{k+1}\right]$ the inequality $t_{0}<t_{k}-\tau \leq s-\tau$ holds. Let $j \leq k$ be a natural number such that $s-\tau \in\left(t_{j}, t_{j+1}\right]$ for $s \in\left[t_{k}, t\right]$. Then by inequality (39) we have

$$
\begin{aligned}
(t & \left.-t_{k}\right)^{1-q}|\Xi(z(t))-\Xi(y(t))| \leq \frac{\left|B_{k}\left(z\left(t_{k}\right)\right)-B_{1}\left(y\left(t_{k}\right)\right)\right|}{\Gamma(q)} \\
& +\frac{\left(t-t_{k}\right)^{1-q}}{\Gamma(q)} \int_{t_{k}}^{t} \frac{|f(s, z(s), z(s-\tau))-f(s, y(s), y(s-\tau))|+\left|H_{k}(s, z)-H_{k}(s, y)\right|}{(t-s)^{1-q}} d s \\
\leq & \frac{C_{k}\left|z\left(t_{k}\right)-y\left(t_{k}\right)\right|}{\Gamma(q)}+\frac{L|| z-\left.y\right|_{k}}{\Gamma(q)} \int_{t_{k}}^{t} \frac{d s}{\left(s-t_{k}\right)^{1-q}(t-s)^{1-q}} \\
& +\frac{M|| z-\left.y\right|_{j}}{\Gamma(q)} \int_{t_{k}}^{t} \frac{d s}{\left(s-\tau-t_{j}\right)^{1-q}(t-s)^{1-q}} \\
& +\frac{1}{\Gamma(q)} \int_{t_{k}}^{t} \frac{\left|H_{k}(s, z)-H_{k}(s, y)\right|}{(t-s)^{1-q}} d s \\
\leq & \left(\frac{C_{k}}{\Gamma(q)\left(t_{k}-t_{k-1}\right)^{1-q}}+\frac{L\left(t-t_{k}\right)^{q} \Gamma(q)}{\Gamma(2 q)}+\frac{M\left(t-t_{k}\right)}{\Gamma(1+q)\left(t_{k}-\tau-t_{0}\right)^{1-q}}\right. \\
& \left.+\Gamma(q)\left(\frac{t-t_{k}}{t-t_{0}}\right)^{1-q} \sum_{i=0}^{k-2} \frac{1}{\left(t_{k}-t_{i}\right)^{q}}+\left(\frac{t-t_{k}}{t-t_{k-1}}\right)^{1-q} \frac{\Gamma(q)}{\left(t_{k}-t_{k-1}\right)^{q}}\right)\|z-y\|_{J} \\
\leq & \left(\frac{C_{k}}{\Gamma(q)\left(t_{k}-t_{k-1}\right)^{1-q}}+\frac{L\left(t_{k+1}-t_{k}\right)^{q} \Gamma(q)}{\Gamma(2 q)}+\frac{M\left(t_{k+1}-t_{k}\right)}{\Gamma(1+q)\left(t_{k}-\tau-t_{0}\right)^{1-q}}\right. \\
& \left.+\left(\frac{t_{k+1}-t_{k}}{t_{k}-t_{0}}\right)^{1-q} \sum_{i=0}^{k-2} \frac{\Gamma(q)}{\left(t_{k}-t_{i}\right)^{q}}+\left(\frac{t_{k+1}-t_{k}}{t_{k}-t_{k-1}}\right)^{1-q} \frac{\Gamma(q)}{\left(t_{k}-t_{k-1}\right)^{q}}\right)\|z-y\|_{J} .
\end{aligned}
$$

Therefore,

$$
\|\Xi(z(t))-\Xi(y(t))\|_{k} \leq \rho\|z-y\|_{J}, \quad t \in\left(t_{k}, t_{k+1}\right] .
$$

Then, $\|\Xi(z(t))-\Xi(y(t))\|_{J} \leq \rho\|z-y\|_{J}$ and the operator $\Xi: P L^{l o c}(J, \mathbb{R}) \rightarrow P L^{l o c}(J, \mathbb{R})$ is a contraction.

Uniqueness. Let $z(t), y(t)$ be two solutions of the IVP (5), (6), (7). According to Theorem 1 each of the functions $z(t)$ and $y(t)$ satisfies the integral presentation (8). Similar to the proofs of inequalities (39), (40) and (41) we obtain that $\|z-y\|_{J} \leq \rho\|z-y\|_{J}$, which proves the uniqueness. 
Example 1. Consider the RL fractional differential equation

$$
{ }_{t_{0}}^{R L} D_{t}^{0.8} x(t)=\frac{0.05 t}{t+1} \sin (x(t))+0.01 \cos (x(t-1)) \text { for } t \in\left(t_{k}, t_{k+1}\right], k=0,1,2,
$$

with the integral form of the initial condition

$$
x(t)=\phi(t), t \in[-1,0], \text { and }\left.{ }_{0} I_{t}^{0.2} x(t)\right|_{t=0}=\phi(0)
$$

and the integral form of the impulsive condition

$$
\left.t_{k} I_{t}^{0.2} x(t)\right|_{t=t_{k}}=(-1)^{k} 0.1 x\left(t_{k}-0\right), \text { for } k=1,2
$$

where $t_{k}=3 k, k=0,1,2, \phi \in C([-1,0], \mathbb{R})$.

In this special case we have $T=9, f(t, x, y)=\frac{0.05 t}{t+1} \sin (x)+0.01 \cos (y), B_{k}(x)=(-1)^{k} 0.1 x, C_{k}=0.1$, $q=0.8, L=0.05, M=0.01, \tau=1, J=[0,9]$.

The condition (32) is reduced to

$$
\frac{0.1}{\Gamma(0.8) 3^{0.2}}+\frac{0.05 * 3^{0.8} \Gamma(0.8)}{\Gamma(1.6)}+\frac{0.03}{\Gamma(1.8) 8^{0.2}}+\left(\frac{3}{9}\right)^{0.2} \frac{\Gamma(0.8)}{9^{0.8}}+\left(\frac{3}{9}\right)^{0.8} \frac{\Gamma(0.8)}{6^{0.8}}+\left(\frac{3}{6}\right)^{0.2} \frac{\Gamma(0.8)}{3^{0.8}}=0.981519<1
$$

According to Theorem 5 the IVP (43)-(45) has a unique solution $x \in P L^{l o c}([0,9], \mathbb{R})$. This solution according to Theorem 1 satisfies

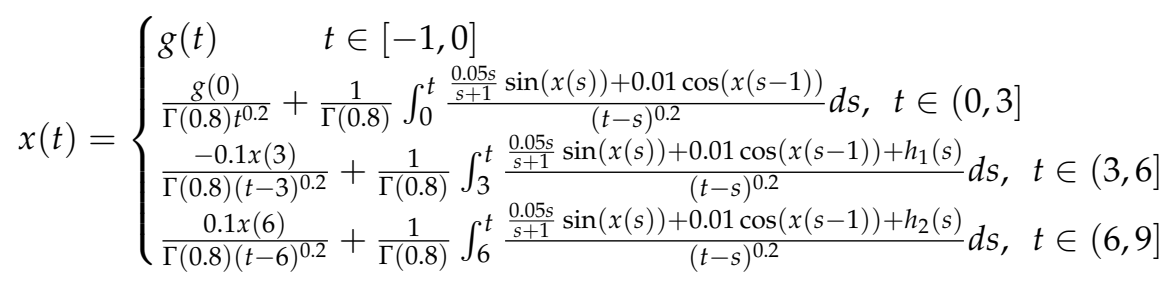

where $h_{1}(t)=\frac{0.8}{\Gamma(0.2)} \int_{0}^{3} \frac{x(s)}{(t-s)^{1.8}} d s$ for $t \in(3,6]$ and $h_{2}(t)=\frac{0.8}{\Gamma(0.2)} \int_{0}^{6} \frac{x(s)}{(t-s)^{1.8}} d s$ for $t \in(6,9]$.

5.2. Changeable Lower Limit of RL Fractional Derivative-Existence for the IVP (22), (6), (7)

Theorem 6. Let the conditions (A1), (A2) be satisfied and the inequality

$$
\rho=\max _{k-1,2, \ldots, N}\left\{\frac{C_{k}}{\Gamma(q)\left(t_{k}-t_{k-1}\right)^{1-q}}+\frac{L\left(t_{k+1}-t_{k}\right)^{q} \Gamma(q)}{\Gamma(2 q)}+\frac{M\left(t_{k+1}-t_{k}\right)}{\Gamma(1+q)\left(t_{k}-\tau-t_{0}\right)^{1-q}}\right\}<1
$$

holds.

Then the IVP (22), (6), (7) has a unique solution $x \in P L^{\text {loc }}(J, \mathbb{R})$.

Proof. Existence. Define the operator $\Xi: P L^{l o c}(J, \mathbb{R}) \rightarrow P L^{l o c}(J, \mathbb{R})$ by

$$
\Xi(x(t))=\left\{\begin{array}{l}
\phi(t) \quad t \in[-\tau, 0] \\
\frac{B_{k}\left(x\left(t_{k}\right)\right)}{\Gamma(q)\left(t-t_{k}\right)^{1-q}}+\frac{1}{\Gamma(q)} \int_{t_{k}}^{t} \frac{f(s, x(s), x(s-\tau))}{(t-s)^{1-q}} d s, \\
\quad \text { for } t \in\left(t_{k}, t_{k+1}\right], k=0,1,2, \ldots, m
\end{array}\right.
$$

where $B_{0}(x) \equiv x$.

Let $z, y \in P L^{l o c}(J, \mathbb{R})$.

For $t \in\left(t_{0}, t_{1}\right]$ as in the proof of Theorem 5 the inequality (40) holds. 
Let $t \in\left(t_{k}, t_{k+1}\right], k=1,2, \ldots, m$. If $t_{k} \leq t_{0}+\tau$ then the inequality (40) holds. Let $t_{k}>t_{0}+\tau$. Then for any $s \in\left[t_{k}, t\right], t \in\left(t_{k}, t_{k+1}\right]$ the inequality $t_{0}<t_{k}-\tau \leq s-\tau$ holds. Let $j \leq k$ be a natural number such that $s-\tau \in\left(t_{j}, t_{j+1}\right]$ for $s \in\left[t_{k}, t\right]$. Then we have

$$
\begin{aligned}
& \left(t-t_{k}\right)^{1-q}|\Xi(z(t))-\Xi(y(t))| \leq \frac{\left|B_{k}\left(z\left(t_{k}\right)\right)-B_{1}\left(y\left(t_{k}\right)\right)\right|}{\Gamma(q)} \\
& +\frac{\left(t-t_{k}\right)^{1-q}}{\Gamma(q)} \int_{t_{k}}^{t} \frac{|f(s, z(s), z(s-\tau))-f(s, y(s), y(s-\tau))|}{(t-s)^{1-q}} d s \\
& \leq \frac{C_{k}\left|z\left(t_{k}\right)-y\left(t_{k}\right)\right|}{\Gamma(q)}+\frac{L|| z-\left.y\right|_{k}}{\Gamma(q)} \int_{t_{k}}^{t} \frac{d s}{\left(s-t_{k}\right)^{1-q}(t-s)^{1-q}} \\
& +\frac{M|| z-y \|_{j}}{\Gamma(q)} \int_{t_{k}}^{t} \frac{d s}{\left(s-\tau-t_{j}\right)^{1-q}(t-s)^{1-q}} \\
& \leq\left(\frac{C_{k}}{\Gamma(q)\left(t_{k}-t_{k-1}\right)^{1-q}}+\frac{L\left(t_{k+1}-t_{k}\right)^{q} \Gamma(q)}{\Gamma(2 q)}+\frac{M\left(t_{k+1}-t_{k}\right)}{\Gamma(1+q)\left(t_{k}-\tau-t_{0}\right)^{1-q}}\right)\|z-y\|_{J} .
\end{aligned}
$$

Therefore, $\|\Xi(z(t))-\Xi(y(t))\|_{J} \leq \rho\|z-y\|_{J}$ and the operator $\Xi$ is a contraction.

Uniqueness. Let $z(t), y(t)$ be two solutions of the IVP (22), (6), (7). According to Theorem 3 each of the functions $z(t)$ and $y(t)$ satisfies the integral presentation (23). Similar to the proof of inequality (47) we obtain that $\|z-y\|_{J} \leq \rho\|z-y\|_{J}$ which proves the uniqueness.

Example 2. Consider the RL fractional differential equation (21) but with cheangable lower limt of the $R L$ fractional derivative, i.e., consider

$$
{ }_{3 k}^{R L} D_{t}^{0.8} x(t)=\frac{0.05 t}{t+1} \sin (x(t))+0.01 \cos (x(t-1) \text { for } t \in(3 k, 3(k+1)], k=0,1,2,
$$

with the integral form of the initial condition (44) and the integral form of the impulsive condition (45). In this special case we have $T=9, f(t, x, y)=\frac{0.05 t}{t+1} \sin (x)+0.01 \cos (y), B_{k}(x)=(-1)^{k} 0.1 x, C_{k}=0.1, q=0.8$, $L=0.05, M=0.01, \tau=1, J=[0,9]$.

The condition (46) is reduced to $\frac{0.1}{\Gamma(0.8) 3^{0.2}}+\frac{0.05 * 3^{0.8} \Gamma(0.8)}{\Gamma(1.6)}+\frac{0.03}{\Gamma(1.8) 8^{0.2}}=0.247094<1$. According to Theorem 6 the IVP (48), (44), (45) has a unique solution $x \in P L^{\text {loc }}([0,9], \mathbb{R})$ (compare with Example 1 ). This solution according to Theorem 3 satisfies

$$
x(t)=\left\{\begin{array}{l}
\phi(t), \quad t \in[-1,0], \\
\frac{\phi(0)}{\Gamma(0.8) t^{0.2}}+\frac{1}{\Gamma(0.8)} \int_{0}^{t} \frac{0.055}{s+1} \sin (x(s))+0.01 \cos (x(s-1)) \\
\frac{-0.1 x(3)}{(-s)^{0.2}} d s, t \in(0,3], \\
\frac{10.8)(t-3)^{0.2}}{\Gamma(0.8)}+\frac{1}{\Gamma(0.8)} \int_{3}^{t} \frac{\frac{0.05 s}{s+1} \sin (x(s))+0.01 \cos (x(s-1))}{(t-s)^{0.2}} d s, t \in(3,6], \\
\frac{0.1 x(6)}{\Gamma(0.8)(t-6)^{0.2}}+\frac{1}{\Gamma(0.8)} \int_{6}^{t} \frac{\frac{0.05 s}{s+1} \sin (x(s))+0.01 \cos (x(s-1))}{(t-s)^{0.2}} d s, \quad t \in(6,9] .
\end{array}\right.
$$

Now consider (48) with changed coefficients, i.e., consider

$$
{ }_{3 k}^{R L} D_{t}^{0.8} x(t)=\frac{0.2 t}{t+1} \sin (x(t))+0.1 \cos (x(t-1) \text { for } t \in(3 k, 3(k+1)], k=0,1,2 .
$$

Then condition (46) is satisfied because $\frac{0.1}{\Gamma(0.8) 3^{0.2}}+\frac{0.2 * 3^{0.8} \Gamma(0.8)}{\Gamma(1.6)}+\frac{0.3}{\Gamma(1.8) 8^{0.2}}=0.90903<1$ and according to Theorem 6 the IVP (49), (44), (45) has a unique solution $x \in P L^{\text {loc }}([0,9], \mathbb{R})$.

Now, consider the equation (49) with fixed lower limit of the RL fractional derivative at 0 , then condition (32) is not satisfied and Theorem 5 cannot be applied to conclude the existence. 
Author Contributions: Conceptualization, R.A., S.H. and D.O.; methodology, R.A., S.H. and D.O.; validation, R.A., S.H. and D.O.; formal analysis, R.A., S.H. and D.O.; investigation, R.A., S.H. and D.O.; writing-original draft preparation, R.A., S.H. and D.O.; writing-review and editing, R.A., S.H. and D.O. All authors have read and agreed to the published version of the manuscript.

Funding: S. Hristova was supported by the Bulgarian National Science Fund under Project KP-06-N32/7.

Conflicts of Interest: The authors declare no conflict of interest.

\section{References}

1. Agarwal, R.; Hristova, S.; O'Regan, D. A survey of Lyapunov functions, stability and impulsive Caputo fractional differential equations. Frac. Calc. Appl. Anal. 2016, 19, 290-318. [CrossRef]

2. Benchohra, M.; Seba, D. Impulsive fractional differential equations in Banach spaces. Electron. J. Qual. Theory Differ. Equ. Spec. Ed. I 2009, 8, 1-14. [CrossRef]

3. Bonanno, G.; Rodriguez-Lopez, R.; Tersian, S. Existence of solutions to boundary value problem for impulsive fractional differential equation. Frac. Calc. Appl. Anal. 2014, 17, 717-744. [CrossRef]

4. Feckan, M.; Zhou, Y.; Wang, J.R. On the concept and existence of solution for impulsive fractional differential equations. Commun. Nonl. Sci. Numer. Simul. 2012, 17, 3050-3060. [CrossRef]

5. Wang, J.R.; Feckan, M.; Zhou, Y. A survey on impulsive fractional differential equations. Frac. Calc. Appl. Anal. 2016, 19, 806-831. [CrossRef]

6. Yukunthorn, W.; Ntouyas, S.K.; Tariboon, J. Impulsive Multiorders Riemann-Liouville Fractional Differential Equations. Discrete Dyn. Nat. Soc. 2015, 2015, 603893. [CrossRef]

7. Chaudharya, R.; Pandey, D.N. Monotone iterative technique for impulsive Riemann-Liouville fractional differential equations. Filomat 2018, 32, 3381-3395. [CrossRef]

8. Liu, Z.; Bin, M. Approximmate controlability of impulsive Riemann-Liouvillve fractional differential equations in Banach space. J. Integral Equ. Appl. 2014, 26, 527-551. [CrossRef]

9. Anguraja, A.; Kanjanadevia, S.; Nieto, J.J. Mild solutions of Riemann-Liouville fractional differential equations with fractional impulses. Nonlin. Anal. Model. Control 2016, 22, 753-764. [CrossRef]

10. Li, M.; Wang, J.R. Representation of solution of a Riemann-Liouville fractional differential equation with pure delay. Appl. Math. Lett. 2018, 85, 118-124. [CrossRef]

11. Li, M.; Wang, J.R. Finite time stability and relative controllability of Riemann-Liouville fractional delay differential equations. Math. Meth. Appl. Sci. 2019, 1-17 [CrossRef]

12. Heymans, N.; Podlubny, I. Physical interpretation of initial conditions for fractional differential equations with Riemann-Liouville fractional derivatives. Rheol. Acta 2006, 45, 765-771. [CrossRef]

13. Diethelm, K. The Analysis of Fractional Differential Equations; Springer: Berlin/Heidelberg, Germany, 2010.

14. Podlubny, I. Fractional Differential Equations; Academic Press: San Diego, CA, USA, 1999.

15. Kilbas, A.A.; Srivastava, H.M.; Trujillo, J.J. Theory and Applications of Fractional Differential Equations; Elsevier: Amsterdam, The Netherlands, 2006.

(C) 2020 by the authors. Licensee MDPI, Basel, Switzerland. This article is an open access article distributed under the terms and conditions of the Creative Commons Attribution (CC BY) license (http://creativecommons.org/licenses/by/4.0/). 